\title{
Yeast adaptation on the substrate straw
}

\author{
Heike Kahr $^{1 *}$, Sara Helmberger ${ }^{1}$, Alexander G. Jäger ${ }^{1}$ \\ ${ }^{1}$ Upper Austria University of Applied Sciences Research and Development Ltd, Campus Wels \\ Stelzhamerstrasse 23, A-4600 Wels, Austria \\ *Tel: +437242 728114170,Fax: +4372427281194170 E-mail: heike.kahr@fh-wels.at
}

\begin{abstract}
Bioethanol production of lignocellulosics is technologically analyzed, but requires further investigations concerning yield optimization and economic efficiency. One important aspect is to obtain an ideal yeast strain for the fermentation process, which should possess the ability of a stable conversion of C5- and C6sugars, resistance/tolerance against inhibitory compounds, temperature, ethanol, sugar and industrial stability. The use of genetically-modified microorganisms is reported. Problems with the stability of the microorganisms and public concerns with regard to/about the use of genetically-modified organisms led us to seek other strategies. For this purpose, several yeast strains were adapted to the mentioned characteristics using specific natural adaptation and systematic selection.

For improved utilization of xylose, several yeast strains have been cultivated on xylose-minimal agar for various generations. Yeast strains have been adapted to grow in ascending concentrations of wheat straw hydrolysate medium. Furthermore, several yeast strains have been bred at increased temperatures, with enhanced ethanol and sugar concentrations.

Some xylose- as well as hydrolysate-adapted yeast strains show an increased fermentative competence. A few of the thermally adapted yeast strains represent an enhanced fermentative capacity at higher temperature $\left(42^{\circ} \mathrm{C}\right)$. Various yeast strains are tolerant concerning 8-12 \% of ethanol and 400-450 g/l of glucose. Some significant improvements concerning ideal yeast strain could have been reached. These improvements offer new possibilities for further optimization.
\end{abstract}

Keywords: Lignocellulosic remnant straw, Ideal yeast strain, Natural adaptation, Systematic selection, Bioethanol

\section{Introduction}

Economic production of bioethanol using lignocellulosic biomass involves quantitative fermentation of cellulosic as well as hemicellulosic fraction, which contains xylose as major sugar component in agricultural remnant materials [1]. By use of the yeast strain Saccharomyces only C6-sugars can be converted to ethanol, but not C5-sugars [2]. If the pentoses were also converted, ethanol yield could be increased significantly.

In the literature, numerous approaches are described where due to genetic engineering the pentose phosphate pathway in yeast cells (especially in Saccharomyces cerevisiae) is modified, so that the yeasts are able to convert xylose into ethanol, reviewed in [3]. Anyway, during longer application in the laboratory, the stability of recombinant yeast strains is not guaranteed, moreover, global acceptance concerning GM-organisms is another serious problem [4]. Therefore, development of non-GM yeast strains, capable of utilizing and converting xylose efficiently for production of bioethanol, using natural selection and breeding would be advantageous. When lignocellulosic biomass (e.g. straw) is pretreated (with Steam Explosion), substances, such as phenols, hydroxymethylfurfural (HMF), furfural and organic acids are generated. Those substances inhibit yeast cells, resulting in a highly constrained growth and ethanol production.

The majority of fermenting yeasts generally have limited osmotolerance and thermotolerance, with an optimum temperature ranging from 30 to $37^{\circ} \mathrm{C}$ [5]. Therefore, the development and use of osmotolerant and thermotolerant yeast strains, capable of growing and fermenting with good yields at temperatures above $40^{\circ} \mathrm{C}$ would be advantageous, especially with regard to 
simultaneous saccharification and fermentation (SSF) [6]. Yeast growth and fermentation at high initial sugar concentrations and high temperature not only minimizes contamination chances, reduces cooling costs, has faster fermentation rates, but also facilitates the attainability of high ethanol concentrations, therefore reducing subsequent distillation costs.

Ethanol is known to act as an inhibitor to yeast cells, inducing loss of cell viability and inhibition of both yeast growth and different transport systems [7]. Thus, ethanol tolerant yeast strains are beneficial, in order to achieve high fermentation efficiency and finally a high yield of ethanol.

Summing up, the ideal yeast strain for bioethanol production of lignocellulosic biomass (e.g. straw) should possess the following characteristics: stable conversion of C5 and C6-sugars, resistance against inhibitory compounds, tolerance concerning temperature, ethanol as well as sugar and industrial stability. In this paper, we demonstrate that various yeast strains could have been adapted to the mentioned characteristics using specific natural adaptation and systematic selection.

\section{Methodology}

\subsection{Microorganisms}

The following microorganisms have been used: Kluyveromyces marxianus (DSM 5418), Kluyveromyces marxianus (DSM 5420), Kluyveromyces thermotolerans (DSM 3434), S.C.BUT8, S.C.BUT4, Candida utilis, S.C.BUT3, Rhodotorula, Kluyveromyces lactis (DSM 4909), osmophilic yeast strain, Saccharomyces cerevisiae, Malaga, white wine yeast strain, Pichia stipitis, Pachysolen tannophilus (DSM 70352) and industrial baker's yeast (S.C.BUT2).

All yeast strains, used for adaptation, were grown in YGC medium at $30^{\circ} \mathrm{C}$ and maintained at $4^{\circ} \mathrm{C}$ on YGC agar plates. Pichia stipitis and Pachysolen tannophilus (DSM 70352) are maintained on Xylose agar plates.

\section{2. $\quad$ Media}

YGC medium (yeast extract $5 \mathrm{~g} / \mathrm{L}$, glucose $20 \mathrm{~g} / \mathrm{L}$, chloramphenicol $0.1 \mathrm{~g} / \mathrm{L}$ ) was routinely used as growth and testing medium. Agar plates have been prepared by adding agar $15 \mathrm{~g} / \mathrm{L}$. Xylose medium (urea $6.4 \mathrm{~g} / \mathrm{L}, \mathrm{KH}_{2} \mathrm{PO}_{4} 1.2 \mathrm{~g} / \mathrm{L}, \mathrm{Na}_{2} \mathrm{HPO}_{4} 0.18 \mathrm{~g} / \mathrm{L}$, yeast extract $10 \mathrm{~g} / \mathrm{L}$, xylose $50 \mathrm{~g} / \mathrm{L}$, chloramphenicol 0,1 g/L) was used as xylose-growth medium. Xylose agar plates have been prepared using yeast extract $10 \mathrm{~g} / \mathrm{L}$, peptone $20 \mathrm{~g} / \mathrm{L}$, xylose $20 \mathrm{~g} / \mathrm{L}$, agar 20 $\mathrm{g} / \mathrm{L}$, chloramphenicol $0.1 \mathrm{~g} / \mathrm{L}$. Xylose minimal-medium (DIFCO ${ }^{\circledR}$ yeast nitrogen base without amino acids $6.7 \mathrm{~g} / \mathrm{L}$, xylose, $50 \mathrm{~g} / \mathrm{L}$, chloramphenicol $0.1 \mathrm{~g} / \mathrm{L}$ ) was used as xyloseadaptation minimal medium [8].

Agar plates have been prepared by adding agar $18 \mathrm{~g} / \mathrm{L}$. Hydrolysate adaptation medium was prepared, similar to [9], by adding xylose $15 \mathrm{~g}$, glucose $30 \mathrm{~g}$, yeast extract $1.5 \mathrm{~g}$, peptone $3 \mathrm{~g}$, $\mathrm{KH}_{2} \mathrm{PO}_{4} 2 \mathrm{~g}$, $\left(\mathrm{NH}_{4}\right)_{2} \mathrm{SO}_{4} 1 \mathrm{~g}$ and $\mathrm{MgSO}_{4}-7 \mathrm{H}_{2} \mathrm{O} 0.5 \mathrm{~g}$ in $1 \mathrm{~L}$ of $10-30 \%$ (dry substance) pre-fabricated straw hydrolysate (described in chapter 2.3), the $\mathrm{pH}$ was adjusted to $5.0 \pm 0.1$. In normal straw hydrolysate (10 \% dry substance), the average xylose yield accounts for about $15 \mathrm{~g} / \mathrm{L}$ and average glucose yield accounts for about $30 \mathrm{~g} / \mathrm{L}$. With increasing hydrolysate concentrations (dry substances of up to $30 \%$ ), glucose and xylose input amounts were reduced in adaptation media. Adaptation media have been finished by sterilely filtrating. 


\subsection{Preparation of wheat straw hydrolysate}

With pretreated, dried and grinded straw, a 10 to $30 \%$ suspension has been produced, using citrate-buffer solution (acetic acid $9.6 \mathrm{~g} / \mathrm{L}, \mathrm{pH} 5.0 \pm 0.1$ ). Suspension has been enzymatically solubilised at a temperature of $50{ }^{\circ} \mathrm{C}$ for 96 hours. After hydrolysis, suspension has been filtered and further used in hydrolysate adaptation media.

\subsection{Determination of sugars, ethanol, organic acids, furans, xylitol}

For precise sugar and ethanol analytics, as well as for determination of HMF, furfural and xylitol, HPLC from Jasco and BioRad AMINEX® HPX 87H with ultra-pure water as eluent, RI detection has been used. For precise organic acids analytics, as well as for lateral validation of sugar-, HMF-, furfural- and xylitol-concentrations, HPLC from Agilent Technologies, Varian Metacarb $87 \mathrm{H}$ with $5 \mathrm{mM} \mathrm{H}_{2} \mathrm{SO}_{4}$ as eluent, UV $210 \mathrm{~nm}$ and RI detection has been used.

\subsection{Xylose-solution for fermentation}

Xylose solution was produced using xylose, $125 \mathrm{~g} / \mathrm{L}$, filled up with citrate buffer solution. After sterilely filtrating, a fermentation nutrient solution has been added. $\mathrm{pH}$-value has been adjusted to 6.3 .

\subsubsection{Fermentation nutrient solution}

Fermentation nutrient solution has been prepared using DIFCO ${ }^{\circledR}$ yeast nitrogen base without amino acids $85 \mathrm{~g} / \mathrm{L}$, urea $113.5 \mathrm{~g} / \mathrm{L}$, peptone $328 \mathrm{~g} / \mathrm{L}$.

\subsection{Glucose-solution for fermentation}

Glucose-solution was produced using glucose $140 \mathrm{~g} / \mathrm{L}$, filled up with citrate buffer solution. After autoclaving, $\left(\mathrm{NH}_{4}\right)_{2} \mathrm{HPO}_{4}, \mathrm{CaCl}_{2}-2 \mathrm{H}_{2} \mathrm{O}, \mathrm{KH}_{2} \mathrm{PO}_{4}$ and $\mathrm{MgSO}_{4}-7 \mathrm{H}_{2} \mathrm{O}$ have been added, $\mathrm{pH}$-value has been adjusted to 4.6.

\subsection{Adaptation of yeast strains on xylose as sole carbon source}

Selected, analyzed yeast strains have been streaked constantly on xylose-containing agar plates (rich medium) for several generations (approx. 15 passages). Those yeast strains have been adapted to grow on Xylose-minimal agar. Yeast strains have been streaked again constantly on that minimal agar for several generations (currently passage 50).

\subsection{Fermentation analyses with xylose-adapted yeast strains}

Fermentation analyses with xylose-adapted yeast strains have been conducted, using xylose liquid medium as yeast growth medium and xylose solution for fermentation, including the fermentation nutrient solution, $\mathrm{pH}$ 6.3. Fermentation has been practised aerobically and anaerobically at $30{ }^{\circ} \mathrm{C}$ for 168 hours. As positive control, not adapted yeast strains have been cultivated in YGC-medium and used in fermentation process with identical parameters.

\subsection{Adaptation of yeast strains on wheat straw hydrolysate}

Selected yeast strains have been cultivated in ascending concentrated hydrolysate adaptation media. Adaptation of yeast strains has been conducted at $30^{\circ} \mathrm{C}$. Simultaneously, several yeast strains were cultivated additionally at higher temperatures (up to $41^{\circ} \mathrm{C}$ ). Therefore, increased inhibitor-resistant yeast strains with a higher temperature tolerance could be achieved. 


\subsection{Fermentation analyses with wheat straw hydrolysate-adapted yeast strains}

Fermentation analyses with hydrolysate-adapted yeast strains have been conducted, using hydrolysate adaptation medium as yeast growth medium. Fermentation has been conducted using pre-fabricated and concentrated straw hydrolysate (60\% of straw dry substance) at $30^{\circ} \mathrm{C}$ for $168 \mathrm{~h}$. As positive control, not adapted yeast strains have been cultivated in YGCmedium and used in fermentation process with identical parameters.

\subsection{Adaptation of yeast strains on increased temperatures}

Several yeast strains have been streaked on YGC agar plates and incubated at increasing temperatures up to $42{ }^{\circ} \mathrm{C}$. Kluyveromyces marxianus has been bred at temperatures up to $45^{\circ} \mathrm{C}$. Thermotolerant yeast strains have been tested additionally in a fermentation approach at higher temperatures.

\subsection{Determination of alcohol and sugar tolerance}

Yeast strains have been grown in YGC medium at $30^{\circ} \mathrm{C}$ for 24 hours and inoculated into YGC medium plus different ethanol concentrations (ranging from 0-13\%). Yeast cell concentrations have been determined using spectrophotometer (OD $600 \mathrm{~nm}$ ) after incubation at $30^{\circ} \mathrm{C}$. Sugar tolerance of yeast strains have been tested after growth in YGC medium, including different concentrations of glucose (up to $450 \mathrm{~g} / \mathrm{L}$ ). Yeast cell concentration was determined using spectrophotometer (OD $600 \mathrm{~nm}$ ) after incubation at $30^{\circ} \mathrm{C}$. Sugar tolerant yeast strains have been tested additionally in a fermentation approach using glucose solution with different glucose concentrations (up to $450 \mathrm{~g} / \mathrm{L}$ ) at $30^{\circ} \mathrm{C}$ for 168 hours.

\section{Results}

\subsection{Fermentation analyses with xylose-adapted yeast strains}

Several xylose-adapted yeast strains (about 50 passages) produced slightly increased ethanol yields, especially during aerobic fermentation process. Using xylose-adapted Pichia stipitis (passage 48) in the aerobic fermentation process, a significant increase in ethanol production with $4.6 \%$ vol. could be observed (table 1 and 2).

Table 1. Ethanol yields after aerobic and anaerobic fermentation of xylose-solution, including fermentation nutrient solution, with non-adapted control yeast Pichia stipitis.

\begin{tabular}{ccccc}
\hline $\begin{array}{c}\text { Control yeast } \\
\text { Pichia stipitis }\end{array}$ & $\begin{array}{c}\text { Fermentation } \\
\text { condition }\end{array}$ & EtOH [\% vol.] & $\begin{array}{c}\text { remaining xylose } \\
{[\mathrm{g} / \mathrm{L}]}\end{array}$ & xylitol [g/L] \\
\hline & aerob & 3,4 & 34,5 & 2,5 \\
& anaerob & 2,1 & 73,4 & 2,4 \\
\hline
\end{tabular}

Table 2. Ethanol yields after aerobic and anaerobic fermentation of xylose-solution, including fermentation nutrient solution, with Pichia stipitis, adapted on xylose as sole carbon source.

\begin{tabular}{ccccc}
\hline $\begin{array}{c}\text { Xylose-adapted } \\
\text { Pichia stipitis }\end{array}$ & $\begin{array}{c}\text { Fermentation } \\
\text { condition }\end{array}$ & EtOH [\% vol.] & $\begin{array}{c}\text { remaining xylose } \\
\text { [g/L] }\end{array}$ & xylitol [g/L] \\
\hline & aerob & 4,6 & 19,7 & 14,4 \\
& anaerob & 4 & 19,1 & 16,2 \\
\hline
\end{tabular}

\subsection{Adaptation of yeast strains on wheat straw hydrolysate medium}

Yeast strains have been adapted successfully to ascending concentrations of wheat straw hydrolysate medium. Both, at $30{ }^{\circ} \mathrm{C}$ and also at increased temperatures (up to $41^{\circ} \mathrm{C}$ ), yeast growth in straw hydrolysate medium is successful. 


\subsection{Fermentation analyses with wheat straw hydrolysate-adapted yeast strains}

Fermentation approaches with hydrolysate-adapted yeast strains have been conducted using concentrated wheat straw hydrolysate (with $60 \%$ of dry substance) (table 3). Fermentation approaches have shown that some adapted yeast strains have increased resistance concerning existent inhibitory compounds (table 4).

Table 3. List of sugars, organic acids and furans, contained in concentrated wheat straw hydrolysate (with $60 \%$ of dry substance).

\begin{tabular}{cc}
\hline $\begin{array}{c}\text { Components of concentrated } \\
\text { hydrolysate }\end{array}$ & {$[\mathrm{g} / \mathrm{L}]$} \\
\hline Glucose & 140.3 \\
Xylose & 55.8 \\
Acetic acid & 9.9 \\
Formic acid & 1.2 \\
Propanoic acid & 1.1 \\
Hydroxymethylfurfural & 0.3 \\
Furfural & 0.2 \\
\hline
\end{tabular}

Table 4. EtOH-yields [\%vol.] after fermentation of concentrated wheat straw hydrolysate, using adapted yeast strains and non-adapted control yeast strains.

\begin{tabular}{cccc}
\hline Adapted yeasts & EtOH [\%vol.] & Control yeasts & EtOH [\%vol.] \\
\hline S.C.BUT3 & 4,8 & S.C.BUT3 & 0,7 \\
S.C.BUT8 & 4,2 & S.C.BUT8 & 2,9 \\
S.C.BUT4 & 4,6 & S.C.BUT4 & 0,7 \\
\hline
\end{tabular}

\subsection{Adaptation of yeast strains on increased temperatures}

Several yeast strains show very good growth on YGC-agar plates at temperatures up to $42^{\circ} \mathrm{C}$, except Saccharomyces cerevisiae, which is only able to grow well at $30^{\circ} \mathrm{C}$. Exclusively, Kluyveromyces marxianus can grow at a temperature up to $45^{\circ} \mathrm{C}$.

\subsection{Analysis of fermentative capacity of different yeast strains at increased temperatures}

Several yeast strains have been tested in a fermentation process, using glucose-solution for fermentation at $40^{\circ} \mathrm{C}$. As positive approach, several yeast strains have also been tested in a fermentation process at $30^{\circ} \mathrm{C}$. Most of the yeast strains produced similar ethanol yields during fermentation at $30^{\circ} \mathrm{C}$ (between 5.3 and $7.2 \%$ vol.) and $40^{\circ} \mathrm{C}$ (between 4.7 and $6.1 \%$ vol.). Only a few yeast strains show a significant collapse concerning fermentative capacity at $40^{\circ} \mathrm{C}$.

\subsection{Determination of alcohol tolerance}

Several yeast strains have been tested concerning growth in YGC medium, including ascending concentrations of ethanol (0-13\%). Yeast cell density has been examined using spectrophotometer (OD $600 \mathrm{~nm}$ ) after incubation at $30^{\circ} \mathrm{C}$. Using $11 \%$ of EtOH in the growth medium, several yeast strains still have good cell density $(60 \%, 74 \%, 58 \%, 61 \%, 60 \%$ and $75 \%$, respectively). With $13 \%$ of EtOH, only one yeast strain has $61 \%$ cell density. Only 3 used yeast strains have low ethanol tolerance (2-4 \% EtOH). 


\subsection{Determination of sugar tolerance and fermentative competence}

After analysis of yeast cell growth in YGC medium with ascending glucose-concentrations, several yeast strains have been tested additionally in a fermentation process, using glucose solution with different concentrations of glucose at $30^{\circ} \mathrm{C}$ for 168 hours. Almost all yeast strains have produced high ethanol yields after fermentation with increased glucoseconcentrations (up to $450 \mathrm{~g} / \mathrm{L}$ glucose). Best results have been reached using two special types of yeast.

\section{Discussion}

Lignocellulosic raw materials contain 5-20 \% of the pentose sugars xylose and arabinose [10]. Microorganisms, able to ferment xylose are found among bacteria, yeast and filamentous fungi [11]. However, natural xylose-fermenting yeast strains, such as Pichia stipitis, Candida shehatae and Pachysolen tannophilus [12] are known to produce low ethanol yields and to reassimilate the produced ethanol [13]. However, Pichia stipitis is known as one of the better yeast strains, able to ferment xylose. During anaerobic fermentation conditions a large portion of xylose is converted to xylitol therefore ethanol yield is accordingly low. Low levels of oxygen are important in the conversion of xylose into ethanol, so that cell viability and NADH balance are maintained.

Agbogbo et al. [14] have tested Pichia stipitis in fermentation approaches on various glucose and xylose mixtures. Maximum ethanol concentration with $100 \%$ of xylose (60 g/L) was 24.3 $\pm 0.34 \mathrm{~g} / \mathrm{L}$, which corresponds to about $3.03 \%$ vol. We have successfully adapted many yeast strains to grow on xylose-minimal medium, according to Attfield and Bell [8], who have demonstrated the development of non-GM yeast strains (Saccharomyces cerevisiae), capable of efficiently growing on xylose.

In our study, xylose-adapted yeast strains, regularly bred on xylose-minimal agar for several generations, produced slightly increased ethanol yields, especially during aerobic fermentation process. Using xylose-adapted Pichia stipitis, a significant increase in ethanol production after aerobic fermentation process of about $35 \%$ can be observed (ethanol yield of $4.6 \%$ vol., see table 1 and 2). Also within anaerobic fermentation process, using xyloseadapted Pichia stipitis, an increase in ethanol production of $90 \%$ can be observed (ethanol yield of $4 \%$ vol., see table 1 and 2). All xylose-adapted yeast strains show good tendency of getting adaped to convert xylose to ethanol, but also with a high production of xylitol as byproduct. However, yeast strains will be further cultivated on xylose-minimal agar, as well as in xylose-liquid medium and fermentative capacity concerning increased xylose conversion will be regularly analyzed.

The most widely studied yeast strains for ethanol fermentation using wheat straw hydrolysate as feedstock are Pichia stipitis [9], Kluyveromyces marxianus [15], native as well as recombinant strains of S.cerevisiae. Best ethanol yields have been obtained with the native non-adapted S.cerevisiae with $31.2 \mathrm{~g} / \mathrm{L}$, which corresponds to about $3.9 \%$ vol. [16]. However, those yeast strains are hindered during fermentation process by inhibitory compounds, generated during pretreatment and hydrolysis of lignocellulosic biomass [17]. Bjorling and Lindman [18] reported, that Pichia stipitis is completely inhibited during fermentation by using a medium, containing $3.9 \mathrm{~g} / \mathrm{L}$ acetic acid, known as an inhibitory component in hydrolysates. In his study, Nigam [9] has improved fermentation performance by adapting xylose-fermenting yeast Pichia stipitis on higher concentrations of acetic acid. The addition of $5 \mathrm{~g} / \mathrm{L}$ acetic acid in hydrolysate medium significantly inhibited sugar utilization and ethanol production. Maximal ethanol yield with inhibitor-adapted yeast culture was $14.5 \mathrm{~g} / \mathrm{L}$, which 
corresponds to about $1.8 \%$ vol. In order to adapt yeast strains to inhibitory compounds in the hydrolysat, several yeast strains, used in our study, have been successfully bred in ascending concentrations of wheat straw hydrolysate medium at $30{ }^{\circ} \mathrm{C}$ and also at increased temperatures (up to $41{ }^{\circ} \mathrm{C}$ ). Fermentation approaches have shown that some hydrolysateadapted yeast strains have increased resistance concerning existent inhibitory compounds. Fermentation has been conducted in pre-fabricated wheat straw hydrolysate, containing among glucose and xylose, $9.9 \mathrm{~g} / \mathrm{L}$ acetic acid, $1.2 \mathrm{~g} / \mathrm{L}$ formic acid, $1.1 \mathrm{~g} / \mathrm{L}$ propanoic acid, $0.3 \mathrm{~g} / \mathrm{L}$ hydroxymethylfurfural and $0.2 \mathrm{~g} / \mathrm{L}$ furfural.

The adapted yeast strain S.C.BUT3 produced an ethanol yield of $4.8 \%$ vol., whereas the not adapted control yeast only $0.7 \%$ vol. Also the adapted yeast S.C.BUT8 yielded an increased ethanol concentration of $4.2 \%$ vol. (not adapted control yeast only $2.9 \%$ vol.). By use of adapted S.C.BUT4, an ethanol yield of $4.6 \% \mathrm{vol}$. could be reached, the not adapted control yeast only produced $0.7 \%$ vol. (see table 4 ).

A serious problem concerning simultaneous saccharification and fermentation (SSF), which appears as a promising alternative among all processes for bioethanol production from lignocellulosic biomass is the different optimum temperatures for saccharification (45 - 50 ${ }^{\circ} \mathrm{C}$ ) and fermentation $\left(25-30^{\circ} \mathrm{C}\right)[6]$. The majority of fermenting yeasts generally have limited osmotolerance and thermotolerance, with an optimum temperature ranging from 30 to $37^{\circ} \mathrm{C}$ [5]. In our study, we could thermally adapt several yeast strains to grow at temperatures up to $42^{\circ} \mathrm{C}$, Kluyveromyces marxianus is able to grow at a temperature of $45^{\circ} \mathrm{C}$. Fermentation approaches at $40^{\circ} \mathrm{C}$ provided good ethanol yields (up to $6.1 \% \mathrm{vol}$ ).

Ethanol is known to be an inhibitor to yeast cells, inducing amongst others loss of cell viability which results in less efficient fermentation process and thus to reduced ethanol yield. The receipt of highly ethanol tolerant yeast mutants is very difficult, isolation requires longterm selection techniques in continuous culture [19]. In our study, numerous yeast strains have been tested concerning growth in YGC medium, including ascending concentrations of ethanol (0-13 \%). Using $11 \%$ of EtOH, several yeast strains have good cell viability. With 13 $\%$ of EtOH, one yeast strain has still $61 \%$ cell density. Fermentation at high initial sugar concentrations provides high ethanol concentrations, which reduces subsequent distillation costs. After analysis of yeast cell growth in YGC medium with ascending glucoseconcentrations, used yeast strains have been tested additionally in a fermentation process, using glucose solution with different concentrations of glucose at $30^{\circ} \mathrm{C}$ for 168 hours. Several yeast strains have produced high ethanol yields after fermentation with increased glucoseconcentrations (up to $450 \mathrm{~g} / \mathrm{L}$ glucose).

\section{Conclusion}

The ideal yeast strain for bioethanol production of lignocellulosic biomass has not been produced yet, because the mentioned requirements could not have been combined completely into one ideal yeast strain. However, some significant improvements with regard to the ideal yeast strain could have been reached. These improvements offer new possibilities for further optimization.

\section{References}

[1] B. C. Saha, Hemicellulose bioconversion, J. Ind. Microbiol. Biotechnol. 30, 2003, pp. 279-291.

[2] B. C. H. Chu and H. Lee, Genetic improvement of Saccharomyces cerevisiae for xylose fermentation, Biotechnology Advances 25, 2007, pp. 425-441. 
[3] B. Hahn-Hägerdal et al., Towards industrial pentose-fermenting yeast strains, Appl. Microbiol. Biotechnol. 74, 2007, pp. 937-953.

[4] I. S. Pretorius, Tailoring wine yeast for the new millennium: novel approaches to the ancient art of winemaking, Yeast 16, 2000, pp. 937-953.

[5] J. B. Kristensen et al., Use of surface active additives in enzymatic hydrolysis of wheat straw, Enzyme Microb. Technol. 40, 2007, pp. 888-95.

[6] H. Jorgensen, J.B. Kristensen, C. Felby, Enzymatic conversion of lignocellulose into fermentable sugars: challenges and opportunities, Biofuels Bioprod. Bioref. 1, 2007, pp. 119-34.

[7] H. Alexandre and C. Charpentier, Biochemical aspects of stuck and sluggish fermentation in grape must, J. Ind. Microbiol. Biotechnol. 20, 1998, pp. 20-27.

[8] P. V. Attfield and P. J. L. Bell, Use of population genetics to derive non-recombinant Saccharomyces cerevisiae strains that grow using xylose as a sole carbon source, FEMS Yeast Res. 6, 2006, pp. 862-868.

[9] J. N. Nigam, Development of xylose-fermenting yeast Pichia stipitis for ethanol production through adaptation on hardwood hemicellulose acid prehydrolysate, Journal of applied microbiology 90, 2001, pp. 208-215.

[10]B. Hahn-Hägerdal et al., Bioethanol - the fuel of tomorrow from the residues of today, Trends in Biotechnology 24, 2006, pp. 549-556.

[11]L. Skoog and B. Hahn-Hägerdal., Xylose fermentation, Enzyme Microb. Technol. 10, 1988, pp. 66-80.

[12]A. K. Chandel et al., Economics and environmental impact of bioethanol production technologies: an appraisal, Biotechnology and Molecular Biology Review 2, 2007, pp. 014-032.

[13]D. Karakashev et al., Anaerobic biotechnological approaches for production of liquid energy carriers from biomass, Biotechnology Letters 29, 2007, pp. 1005-1012.

[14]F. K. Agbogbo et al., Fermentation of glucose/xylose mixtures using Pichia stipitis, Process Biochemistry 41, 2006, pp. 2333-2336.

[15]E. Tomás-Pejó et al., Bioethanol production from wheat straw by the thermotolerant yeast Kluyveromyces marxianus CECT 10875 in a simultaneous saccharification and fermentation fed-batch process, Fuel 88, 2009, pp. 2142-2147.

[16]H. Jorgensen, Effect of nutrients on fermentation of pretreated wheat straw at very high dry matter content by Saccharomyces cerevisiae, Applied biochemistry and Biotechnology 153, 2009, pp. 44-57.

[17]B. Hahn-Hägerdal et al., An interlaboratory comparison of the performance of ethanolproducing microorganisms in a xylose-rich acid hydrolysate, Appl. Microbiol. Biotechnol. 41, 1994, pp. 62-72.

[18]T. Bjorling and B. Lindman, Evaluation of xylose-fermenting yeasts for ethanol production from spent sulfite liquor, Enzyme and Microbial. Technology 11, 1989, pp. 240-246

[19] S. W. Brown and S. G. Oliver, Isolation of ethanoltolerant mutants of yeast by continuous selection, Eur. J. Appl. Microbiol. Biotechnol. 16, 1983, pp. 116-122 